\title{
RATE OF ESCAPE OF RANDOM WALKS ON FREE PRODUCTS
}

\author{
LORENZ A. GILCH
}

(Received 23 June 2005; revised 24 April 2006)

Communicated by V. Stefanov

\begin{abstract}
Suppose we are given the free product $V$ of a finite family of finite or countable sets $\left(V_{i}\right)_{i \in \mathscr{I}}$ and probability measures on each $V_{i}$, which govern random walks on it. We consider a transient random walk on the free product arising naturally from the random walks on the $V_{i}$. We prove the existence of the rate of escape with respect to the block length, that is, the speed at which the random walk escapes to infinity, and furthermore we compute formulae for it. For this purpose, we present three different techniques providing three different, equivalent formulae.
\end{abstract}

2000 Mathematics subject classification: primary 60G50; secondary 20E06, 60B 15.

Keywords and phrases: random walks, free products, rate of escape.

\section{Introduction}

Consider a transient Markov chain $\left(Z_{n}\right)_{n \in N_{0}}$ on a state space $V$ and a suitable length function $l$ on $V$ representing a 'word length' with respect to the starting point of the Markov chain. We are interested in whether the sequence of random variables $l\left(Z_{n}\right) / n$ converges almost surely to a constant, and if so, to compute this constant. If the limit exists, it is called the rate of escape, or the drift with respect to $l$. In this paper, we study this question for random walks on general free products.

To outline some background material, on the $d$-dimensional grid $\mathbb{Z}^{d}$, where $d \geq 1$, random walks can be described by the sum of $n$ independent and identically distributed random variables, the increments of $n$ steps. By the weak law of large numbers, the limit $\lim _{n \rightarrow \infty}\left|Z_{n}\right| / n$, where $|\cdot|$ is the distance on the grid to the starting point of the random walk, exists almost surely. Furthermore, this limit is positive if the increments have non-zero mean vector.

(C) 2007 Australian Mathematical Society 1446-7887/07\$A2.00+0.00 
It is well-known that the rate of escape exists also for transitive random walks on finitely generated groups, where the random walks arise from probability measures on the group elements. This follows from Kingman's subadditive ergodic theorem; see Kingman [9], Derriennic [3] and Guivarc'h [7]. If $l$ is the metric of the Cayley

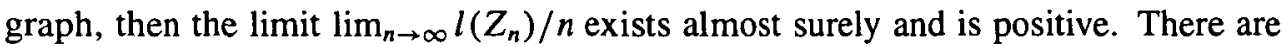
many detailed results for random walks on groups and wreath products: Mairesse [13] computed a explicit formula in terms of the unique solution of a system of polynomial equations for the rate of escape of random walks on the braid group. Lyons, Pemantle and Peres [11] gave a lower bound for the rate of escape of inward-biased random walks on lamplighter groups. Dyubina [4] proved that the drift on the wreath product $A<\mathbb{Z} / 2$ is zero, where $A$ is a finitely generated group. Erschler [5] investigated asymptotics of the drift of symmetric random walks on finitely generated groups. An important link between drifts and harmonic analysis was obtained by Varopoulos [19]. He proved that for symmetric finite range random walks on groups the existence of non-trivial bounded harmonic functions is equivalent to a non-zero rate of escape. This leads to a link between the rate of escape and the entropy of random walks, compare for example with Kaimanovich and Vershik [8] and Erschler [5]. The rate of escape has also been studied on trees: Cartwright, Kaimanovich and Woess [1] investigated the boundary of homogeneous trees and the drift on them. Nagnibeda and Woess [16, Section 5] proved that the rate of escape of random walks on trees with finitely many cone types is non-zero and gave a formula for it.

For a restricted class of free products of finite groups, Mairesse [12] and Mairesse and Mathéus [14] developed a specific technique for computation of the above limit with respect to the word length. These papers were the starting point for the present investigation of arbitrary free products. We consider the free product of finitely many sets, on which Markov chains are given, and construct in a natural way a random walk on the free product. The techniques we use for rewriting probability generating functions in terms of functions on the factors of the free product were introduced independently and simultaneously by Cartwright and Soardi [2], Woess [22], Voiculescu [20] and McLaughlin [15].

Our aim is to show the existence of the above rate of escape $\ell$ with respect to the word length, and also to compute formulae for it. For this purpose, we present three different, equivalent formulae for $\ell$ using three different techniques. In Section 3 we prove existence and a formula for $\ell$ by purely probabilistic reasoning. In Section 4 we compute the proposed limit using double generating functions and applying a theorem of Sawyer and Steger [17, Theorem 2.2]. The third approach for the computation of $\ell$ in Section 5 works only for free products of finitely generated groups and is based on a technique that was used by Ledrappier [10] and Furstenberg [6]. Section 6 presents sample computations and in Section 7 we give additional remarks about extensions of these techniques to further results. 


\section{Free products}

2.1. Free products and random walks Let $\mathscr{I}:=\{1, \ldots, r\}, r \geq 2$. Consider $r$ random walks with transition matrices $P_{i}$ on pairwise disjoint finite or countable state spaces $V_{i}$, where $i \in \mathscr{I}$. The corresponding single and $n$-step transition probabilities are denoted by $p_{i}(x, y)$ and $p_{i}^{(n)}(x, y)$, where $x, y \in V_{i}$. For every $i \in \mathscr{I}$ we select an element $o_{i}$ of $V_{i}$ as the 'root'. To help visualize this, we think of graphs $X_{i}$ with vertex sets $V_{i}$ and roots $o_{i}$ such that there is an oriented edge $x \rightarrow y$ if and only if $p_{i}(x, y)>0$. Furthermore, we shall assume that for every $i \in \mathscr{I}$ and every $x \in V_{i}$ there is an $n \in \mathbb{N}_{0}$ such that $p_{i}^{(n)}\left(o_{i}, x\right)>0$. For the sake of simplicity we assume $p_{i}(x, x)=0$ for every $i \in \mathscr{I}$ and $x \in V_{i}$.

Let $V_{i}^{\times}:=V_{i} \backslash\left\{o_{i}\right\}$ for every $i \in \mathscr{I}$. The next step is the construction of a new Markov chain on the free product $V:=V_{1} * \cdots * V_{r}$, the set of 'words'

$$
x=x_{1} x_{2} \ldots x_{n}
$$

with letters, also called blocks, from the sets $V_{i}^{\times}$such that no two successive letters come from the same $V_{i}$. The empty word $o$ describes the root of $V$. If $u=u_{1} \ldots u_{m} \in V$ and $v=v_{1} \ldots v_{n} \in V$ with $u_{m} \in V_{i}$ and $v_{1} \notin V_{i}$, then $u v$ stands for their concatenation as words. We also define $v o=o v=v$ for all $v \in V$. We regard each $V_{i}$ as a subset of $V$, identifying each $o_{i}$ with $o$.

We lift $P_{i}$ to a transition matrix $\bar{P}_{i}$ on $V:$ if $z \in V$ is $o$ or has last letter not in $V_{i}$, and if $v, w \in V_{i}$, then we set $\bar{p}_{i}(z v, z w):=p_{i}(v, w)$. Otherwise we set $\bar{p}_{i}(x, y):=0$. We choose $0<\alpha_{1}, \ldots, \alpha_{r} \in \mathbb{R}$ with $\sum_{i \in \mathscr{I}} \alpha_{i}=1$. Then we obtain a new transition matrix on $V$ given by

$$
P=\sum_{i \in \mathscr{I}} \alpha_{i} \bar{P}_{i}
$$

The random walk governed by $P$ is described by the sequence of random variables $\left(Z_{n}\right)_{n \in \mathrm{N}_{0}}$. The associated single and $n$-step transition probabilities are denoted by $p(x, y)$ and $p^{(n)}(x, y)$ for $x, y \in V$.

Let $x=x_{1} \ldots x_{m} \in V \backslash\{o\}$. The type $\tau(x)$ of $x$ is defined to be $i$ if $x_{m} \in V_{i}$. The block length $\ell(x)$ of $x$ is defined to be $m$. We also set $\tau(o):=0$ and $\ell(o):=0$. We want to show existence of the $P$-almost sure limit $\ell=\lim _{n \rightarrow \infty} \ell\left(Z_{n}\right) / n$, the rate of escape with respect to the block length, and to present formulae for it. Let $k \in \mathbb{N}$. Then $x^{(k)}:=x_{1} \ldots x_{j}$, where $j=\min \{k, m\}$, is the truncation at length $k$. We also write $x^{\perp}:=x_{1} \ldots x_{m-1}$ for the truncation at length $\ell(x)-1$, if $x \neq o$. Furthermore we denote by $\tilde{x}:=x_{m}$ the terminal block of $x$, if $m>0$, and set $\tilde{o}:=0$. The cone rooted at $x$ is the set

$$
C_{x}:=\left\{y \in V \mid y^{(m)}=x\right\} \subseteq V .
$$


If $y \in V_{i}$, then the set of successors of $y$ is given by

$$
\mathscr{S}(y):=\left\{w \in V_{i} \mid p_{i}(y, w)>0\right\}
$$

and the set of predecessors by

$$
\mathscr{P}(y):=\left\{w \in V_{i} \mid p_{i}(w, y)>0\right\} .
$$

We now introduce some probability generating functions. For this purpose, let $T_{y}:=\min \left\{k \geq 0 \mid Z_{k}=y\right\}\left(S_{y}:=\min \left\{k>0 \mid Z_{k}=y\right\}\right)$ be the stopping time of the first visit (the first return) to $y \in V$. Denote by $\mathbb{P}_{x}$ the probability measure on $V^{N_{0}}$ that governs the random walk starting at $x \in V$. For $z \in \mathbb{C}$ and $x, y \in V$, let

$$
\begin{aligned}
& G(x, y \mid z)=\sum_{n \geq 0} p^{(n)}(x, y) z^{n}, \quad F(x, y \mid z)=\sum_{n \geq 0} \mathbb{P}_{x}\left[T_{y}=n\right] z^{n}, \\
& U(x, y \mid z)=\sum_{n \geq 1} \mathbb{P}_{x}\left[S_{y}=n\right] z^{n}, \\
& L(x, y \mid z)=\sum_{n \geq 0} \mathbb{P}_{x}\left[S_{y}>n, Z_{n}=y\right] z^{n} .
\end{aligned}
$$

The analogous functions for the random walks on the single factors $V_{i}$ are denoted by $G_{i}(u, v \mid z), F_{i}(u, v \mid z)$ and $L_{i}(u, v \mid z)$, where $u, v \in V_{i}$. We make the basic assumption that the radius of convergence of $G(o, o \mid z)$ is greater than 1 , which implies transience of our random walk on $V$. Thus, we may exclude the case $r=2=\left|V_{1}\right|=\left|V_{2}\right|$. This convergence property is fulfilled if each $p_{i}$ is reversible and due to non-amenability also for random walks on free products of finitely generated groups, where the $P_{i}$ depend only on probability measures on the single groups. Note that for $|z|<1$, $\sum_{y \in V_{i}} G_{i}(x, y \mid z)=1 /(1-z)$ for every $i \in \mathscr{I}$ and all $x \in V_{i}$. This will be used several times in the sequel.

Recall the following equations.

LEMMA 2.1. Let $x, y \in V, w \in V \backslash\{$ o $\}$ such that $w^{(1)} \notin V_{\tau(x)}$, and $z \in \mathbb{C}$. Then

(i) $G(x, x \mid z)=1 /(1-U(x, x \mid z))$,

(ii) $G(x, y \mid z)=F(x, y \mid z) G(y, y \mid z)$,

(iii) $G(x, y \mid z)=G(x, x \mid z) L(x, y \mid z)$,

(iv) $F(o, x w \mid z)=F(o, x \mid z) F(x, x w \mid z)$,

(v) $L(o, x w \mid z)=L(o, x \mid z) L(x, x w \mid z)$,

(vi) $L(x, x w \mid z)=L(o, w \mid z)$.

Equations analogous to (i), (ii) and (iii) hold for the generating functions on the single factors $V_{i}$ for every $i \in \mathscr{I}$. 
ProOF. For (i) and (ii) see Woess [23, Lemma 1.13]. Equation (iii) is obtained by conditioning with respect to the last visit at $x$ before finally walking to $y$. Equations (iv) and (v) are obtained by conditioning with respect to the first (last) visit at $x$, which must be visited before finally walking to $x w$. Equation (vi) holds, since by the treelike structure of the free product the probability of walking from $x$ to $x w$ in $n$ steps without returning to $x$ is the same as walking from $o$ to $w$ in $n$ steps without returning to $o$.

We now explain the correspondence between $F(x, y \mid z)$ and $F_{i}(x, y \mid z)$, and $L(x, y \mid z)$ and $L_{i}(x, y \mid z)$. Therefore define for $i \in \mathscr{I}$ and $z \in \mathbb{C}$

$$
\bar{H}_{i}(z):=\sum_{n=2}^{\infty} \mathbb{P}_{o}\left[S_{o}=n, Z_{1} \notin V_{i}\right] z^{n} \quad \text { and } \quad \xi_{i}(z):=\frac{\alpha_{i} z}{1-\bar{H}_{i}(z)} ;
$$

see Woess [23, Proposition 9.18]. Note that $\bar{H}_{i}(1)$ is the probability of starting at some $x \in V_{i}$ and returning to the same $x$ without having visited a neighbour $y \in \mathscr{S}(x)$ of $x$. Similarly $\xi_{i}(1)$ is the probability of starting at some $x \in V_{i}$ and at some time visiting a neighbour $y \in \mathscr{S}(x)$. For positive $z$ the functions $\bar{H}_{i}(z)$ and $\xi_{i}(z)$ are strictly increasing inside their radii of convergence, which are greater than 1 .

LEMMA 2.2. Let $i \in \mathscr{I}, x, y \in V_{i}$ and $z \in \mathbb{C}$. Then

(i) $F(x, y \mid z)=F_{i}\left(x, y \mid \xi_{i}(z)\right)$,

(ii) $L(x, y \mid z)=L_{i}\left(x, y \mid \xi_{i}(z)\right)$.

For the proof of (i) see Woess [23, Proposition 9.18 (c)]. Statement (ii) is proved analogously.

LEMMA 2.3. $\xi_{i}:=\xi_{i}(1)<1$ for all $i \in \mathscr{I}$.

PROOF. Let $H_{i}(z):=U(o, o \mid z)-\bar{H}_{i}(z)$. By transience we have

$$
U(o, o \mid 1)=\sum_{i \in \mathscr{I}} H_{i}(1)<1 .
$$

Furthermore

$$
H_{i}(1)=\alpha_{i} \sum_{s \in \mathcal{S}\left(o_{i}\right)} p_{i}\left(o_{i}, s\right) \underbrace{F(s, o \mid 1)}_{\leq 1} \leq \alpha_{i}
$$

Hence,

$$
\xi_{i}=\frac{\alpha_{i}}{1-\sum_{j \in \mathcal{O} \backslash(i\}} H_{j}(1)} \leq \frac{\alpha_{i}}{1-\sum_{j \in \mathscr{T} \backslash(i\}} \alpha_{j}}=\frac{\alpha_{i}}{1-\left(1-\alpha_{i}\right)}=1
$$

Observe that $\xi_{j}<1$ for all $j \in \mathscr{I} \backslash\{i\}$, if $H_{i}(1)<\alpha_{i}$ for some $i \in \mathscr{I}$. Assume $H_{i}(1)=\alpha_{i}$ for some $i \in \mathscr{I}$. Then $\bar{H}_{i}(1)=U(o, o \mid 1)-H_{i}(1)<1-\alpha_{i}$, and thus 
there is $j \in \mathscr{I} \backslash\{i\}$ such that $H_{j}(1)<\alpha_{j}$. Thus $\xi_{i}<1$. Since

$$
H_{i}(1)=\alpha_{i} \sum_{s \in \mathscr{S}\left(o_{i}\right)} p_{i}\left(o_{i}, s\right) F(s, o \mid 1)=\alpha_{i},
$$

we have $F(s, o \mid 1)=1$ for all $s \in \mathscr{S}\left(o_{i}\right)$. However now we obtain the contradiction

$$
F(s, o \mid 1)=F_{i}\left(s, o_{i} \mid \xi_{i}\right)<1,
$$

as $\xi_{i}<1$ and $F_{i}\left(s, o_{i} \mid x\right), 0 \leq x \in \mathbb{R}$, is strictly increasing with $F_{i}\left(s, o_{i} \mid 1\right) \leq 1$.

2.2. Limit of the random walk As we have assumed transience for the random walk on $V$, the random walk escapes to infinity in the sense that almost surely every finite $A \subseteq V$ is visited only finitely often. We shall now investigate the route of the escape of the random walk on $V$, which provides the main tool for further computations. Define for $x \in V, i \in \mathscr{I}$ with $\tau(x) \neq i$ and $S \subseteq V_{i}$ the set $x S:=\{x y \mid y \in S\}$.

LEMMA 2.4. If $x \in V$ and $\tau(x) \neq i$, then

$$
\mathbb{P}_{o}\left[Z_{n} \in x V_{i} \text { holds for infinitely many } n\right]=0 \text {. }
$$

ProOF. By Lemmas 2.1 and 2.2,

$$
\begin{aligned}
\sum_{n \geq 0} \mathbb{P}_{o}\left[Z_{n} \in x V_{i}\right] & =\sum_{y \in V_{i}} G(o, x y \mid 1) \\
& =\sum_{y \in V_{i}} G(o, o \mid 1) L(o, x \mid 1) L_{i}\left(o_{i}, y \mid \xi_{i}\right) \\
& =G(o, o \mid 1) L(o, x \mid 1) \sum_{y \in V_{i}} \frac{G_{i}\left(o, y \mid \xi_{i}\right)}{G_{i}\left(o_{i}, o_{i} \mid \xi_{i}\right)} \\
& =\frac{G(o, o \mid 1) L(o, x \mid 1)}{G_{i}\left(o_{i}, o_{i} \mid \xi_{i}\right)} \frac{1}{1-\xi_{i}}<\infty .
\end{aligned}
$$

The Borel-Cantelli lemma implies the proposed statement.

Now we are able to specify how the random walk on $V$ escapes to infinity. Let $V_{\infty}$ denote the set of infinite words $x_{1} x_{2} \ldots$ in which each of the letters $x_{j}$ belongs to $\bigcup_{i \in \mathscr{I}} V_{i}^{\times}$, no consecutive letters come from the same $V_{i}^{\times}$, and infinitely many letters come from each $V_{i}^{\times}$.

PROPOSITION 2.5. $\ell\left(Z_{n}\right)$ tends to infinity $\mathbb{P}_{o}$-a.s., as $n \rightarrow \infty$. Furthermore, there exists a $V_{\infty}$-valued random variable $Z_{\infty}$, such that

$$
\lim _{n \rightarrow \infty} Z_{n}=Z_{\infty} \quad \mathbb{P}_{o} \text {-a.s., }
$$

with convergence in the sense that the length of the common prefix of $Z_{n}$ and $Z_{\infty}$ tends to infinity. 
PROOF. We prove by induction that for each $m \in \mathbb{N}$ there is almost surely an $n_{m} \in \mathbb{N}$ with $\ell\left(Z_{n_{m}}\right)=m$ and $\ell\left(Z_{n}\right)>m$ for all $n>n_{m}$. By Lemma 2.4 , the random walk visits the state set $\bigcup_{i \in \mathscr{I}} V_{i}$ finitely often $\mathbb{P}_{o}$-a.s. Therefore there is a maximal index $n_{1} \in \mathbb{N}$ such that $Z_{n_{1}} \in \bigcup_{i \in \mathscr{I}} V_{i} \mathbb{P}_{o}$-a.s. and $Z_{n} \notin \bigcup_{i \in \mathscr{I}} V_{i}$ for all $n>n_{1}$. Thus $\ell\left(Z_{n_{1}}\right)=1$ and $\ell\left(Z_{n}\right)>1$ for all $n>n_{1}$.

Assume now that $\ell\left(Z_{n_{m}}\right)=m$ and $\ell\left(Z_{n}\right)>m$ for all $n>n_{m}$, and let $\mathscr{I}^{\prime}:=\mathscr{I} \backslash\left\{\tau\left(Z_{n_{m}}\right)\right\}$. Again, by Lemma 2.4 , the random walk visits the state set $\bigcup_{i \in \mathscr{I}}, Z_{n_{m}} V_{i}$ finitely often $\mathbb{P}_{o}$-a.s. Then there is a maximal $n_{m+1} \in \mathbb{N}$ such that $Z_{n_{m+1}} \in \bigcup_{i \in \mathscr{I}}, Z_{n_{m}} V_{i}$ and $Z_{n} \notin \bigcup_{i \in \mathscr{I}}, Z_{n_{m}} V_{i}$ for all $n>n_{m+1}$. Thus $\ell\left(Z_{n_{m+1}}\right)=m+1$ and $\ell\left(Z_{n}\right)>m+1$ for all $n>n_{m+1}$. Thus $\ell\left(Z_{n}\right)$ tends to infinity, as $n \rightarrow \infty$.

It is clear that the sequence $\left(Z_{n}\right)_{n \in \mathbb{N}}$ converges to an infinite word in $V_{\infty}$ with $Z_{\infty}^{(m)}=Z_{n_{m}}$ for all $m \in \mathbb{N}$.

\section{Exit time technique}

In this section we investigate the random walk on $V$ in detail, prove the existence of $\ell$ and derive a formula for it. The following technique was motivated by Nagnibeda and Woess [16, Section 5]. Let $k \in \mathbb{N}$. The exit time with respect to the block length $k$ is $\mathbf{e}_{k}:=\min \left\{m \in \mathbb{N} \mid \forall n \geq m: Z_{n}^{(k)}\right.$ constant $\}$. In particular, $\mathbf{e}_{0}=0$. The exit point with respect to the block length $k$ is $W_{k}:=Z_{\mathbf{e}_{k}}$. Thus, $\mathbf{e}_{k}$ is the first instant from which point the first $k$ blocks remain constant, and $W_{k}=x=x_{1} \ldots x_{k}$ if and only if, at time $\mathbf{e}_{k}-1$, the random walk is at state $x_{1} \ldots x_{k-1} s$ with some $s \in \mathscr{P}\left(x_{k}\right)$, at time $\mathbf{e}_{k}$ at state $x$, and thereafter remains in the cone $C_{x}$.

As $Z_{n}$ converges almost surely to a random variable $Z_{\infty}$ with values in $V_{\infty}$, we have $\mathbf{e}_{k} \rightarrow \infty$ as $k \rightarrow \infty$, almost surely. The $k$-th increment is $\mathbf{i}_{k}:=\mathbf{e}_{k}-\mathbf{e}_{k-1}$ and the maximal temporary exit time at time $n$ is defined as $\mathbf{k}(n):=\max \left\{k \in \mathbb{N}_{0} \mid \mathbf{e}_{k} \leq n\right\}$. Define for $i \in \mathscr{I}, y \in V$ and $n \in \mathbb{N}_{0}$

$$
k_{i}^{(n)}(o, y):=\mathbb{P}_{o}\left[\forall j \in\{0, \ldots, n\}: Z_{j} \notin V_{i}^{\times}, Z_{n}=y\right]
$$

and the corresponding generating function

$$
K_{i}(o, y \mid z):=\sum_{n=0}^{\infty} k_{i}^{(n)}(o, y) z^{n}=\sum_{n \geq 0} \bar{H}_{i}(z)^{n} L(o, y \mid z)=\frac{L(o, y \mid z)}{1-\bar{H}_{i}(z)} .
$$

PROPOSITION 3.1. $\left(W_{k}, \mathbf{i}_{k}\right)_{k \in N}$ is a Markov chain with transition probabilities

$$
\begin{array}{r}
\mathbb{P}_{o}\left[W_{k+1}=w_{k+1}, \mathbf{i}_{k+1}=n_{k+1} \mid W_{k}=w_{k}, \mathbf{i}_{k}=n_{k}\right] \\
=\frac{1-\xi_{\tau\left(w_{k+1}\right)}}{1-\xi_{\tau\left(w_{k}\right)}} \sum_{s \in \mathscr{P}(y)}\left[k_{\tau\left(w_{k}\right)}^{\left(n_{k+1}-1\right)}(o, s) p(s, y)\right]
\end{array}
$$

for $n_{k}, n_{k+1} \in \mathbb{N}, w_{k}=x_{1} \ldots x_{k} \in V, w_{k+1}=w_{k} y$, where $y \in \bigcup_{i \in \mathscr{I} \backslash\left\{\tau\left(w_{k}\right)\right\}} V_{i}^{\times}$. 
PROOF. Define $\bar{V}_{i}=\bigcup_{j \in \mathscr{I} \backslash\{i\}} V_{j}^{\times}$. For $2 \leq i \leq k$, let $w_{0}=o, w_{1}=g_{1} \in \bigcup_{i \in \mathscr{I}} V_{i}^{\times}$and $w_{i}=w_{i-1} g_{i}$ with $g_{i} \in \bar{V}_{\tau\left(w_{i-1}\right)}$.

For $i \in\{1, \ldots, k\}$ the inclusion $\left[W_{i+1}=w_{i+1}\right] \subseteq\left[W_{i}=w_{i}\right]$ holds, as $w_{i+1}$ determines the element $w_{i}$ uniquely. Let $n_{1}, \ldots, n_{k+1} \in \mathbb{N}$ and write for $m \in\{k, k+1\}$

$$
\left[W_{1}^{m}=w_{1}^{m}, \mathbf{i}_{1}^{m}=n_{1}^{m}\right]:=\left[\forall j \in\{1, \ldots, m\}: W_{j}=w_{j}, \mathbf{i}_{j}=n_{j}\right] .
$$

This event can be described as follows: start at $o$, walk in $n_{1}-1$ steps to a predecessor of $w_{1}$ inside $V_{\tau\left(w_{1}\right)}$, then walk to $w_{1}$. Then stay inside $C_{w_{1}}$ and walk in $n_{2}-1$ steps to a vertex in $w_{1} \mathscr{P}\left(g_{2}\right)$, from there to $w_{2}$, and so on. More formally we obtain, writing $n_{1}^{s}=\sum_{t=1}^{s} n_{t}$,

$$
\begin{aligned}
\mathbb{P}_{o}\left[W_{1}^{k}\right. & \left.=w_{1}^{k}, \mathbf{i}_{1}^{k}=n_{1}^{k}\right] \\
& =\mathbb{P}_{o}\left[\begin{array}{c}
\forall \lambda \in\{0, \ldots, k-1\} \forall j \in\left\{1, \ldots, n_{\lambda}-1\right\}: \\
Z_{n_{1}^{\lambda}+j} \in C_{w_{\lambda}}, Z_{n_{1}^{\lambda+1}-1} \in w_{\lambda} \mathscr{P}\left(g_{\lambda+1}\right), Z_{n_{1}^{\lambda+1}}=w_{\lambda+1}
\end{array}\right]\left(1-\xi_{\tau\left(w_{k}\right)}\right) .
\end{aligned}
$$

Analogously,

$$
\begin{aligned}
\mathbb{P}_{o}\left[W_{1}^{k+1}=w_{1}^{k+1}, \mathbf{i}_{1}^{k+1}=n_{1}^{k+1}\right] \\
=\mathbb{P}_{o}\left[\begin{array}{c}
\forall \lambda \in\{0, \ldots, k-1\} \forall j \in\left\{1, \ldots, n_{\lambda}-1\right\}: \\
Z_{n_{1}^{\lambda}+j} \in C_{w_{\lambda}}, Z_{n_{1}^{\lambda+1}-1} \in w_{\lambda} \mathscr{P}\left(g_{\lambda+1}\right), Z_{n_{1}^{\lambda+1}}=w_{\lambda+1}
\end{array}\right] \\
\quad \times \mathbb{P}_{w_{k}}\left[\begin{array}{c}
\forall j \in\left\{1, \ldots, n_{k+1}-2\right\}: Z_{j} \in C_{w_{k}}, \\
Z_{n_{k+1}-1} \in w_{k} \mathscr{P}\left(g_{k+1}\right), Z_{n_{k+1}}=w_{k+1}
\end{array}\right]\left(1-\xi_{\tau\left(w_{k+1}\right)}\right) .
\end{aligned}
$$

Thus we obtain the conditional probabilities

$$
\begin{aligned}
& \mathbb{P}_{o}\left[W_{k+1}=w_{k+1}, \mathbf{i}_{k+1}=n_{k+1} \mid W_{1}^{k}=w_{1}^{k}, \mathbf{i}_{1}^{k}=n_{1}^{k}\right] \\
& =\frac{1-\xi_{\tau\left(w_{k+1}\right)}}{1-\xi_{\tau\left(w_{k}\right)}} \mathbb{P}_{w_{k}}\left[\begin{array}{l}
\forall j \in\left\{1, \ldots, n_{k+1}-2\right\}: Z_{j} \in C_{w_{k}}, \\
Z_{n_{k+1}-1} \in w_{k} \mathscr{P}\left(g_{k+1}\right), Z_{n_{k+1}}=w_{k+1}
\end{array}\right] \\
& =\frac{1-\xi_{\tau\left(w_{k+1}\right)}}{1-\xi_{\tau\left(w_{k}\right)}} \sum_{s \in \mathscr{P}(y)}\left[k_{\tau\left(w_{k}\right)}^{\left(n_{k+1}-1\right)}(o, s) p(s, y)\right] .
\end{aligned}
$$

The transition probabilities of the stochastic process $\left(W_{k}, \mathbf{i}_{k}\right)_{k \in \mathbb{N}}$ depend only on $\tau_{k}:=\tau\left(W_{k}\right), \tau\left(W_{k+1}\right)$ and $\widetilde{W}_{k+1}$. Hence, the stochastic process $\left(\widetilde{W}_{k}, \mathbf{i}_{k}, \tau_{k}\right)_{k \in \mathbb{N}}$ is an irreducible Markov chain on the state space

$$
\mathscr{A}:=\left\{(y, n, j) \mid j \in \mathscr{I}, y \in V_{j}^{\times}, n \in \mathbb{N}, \exists i \in \mathscr{I}, s \in \mathscr{P}(y): k_{i}^{(n-1)}(o, s)>0\right\}
$$

with transition probabilities

$$
q((x, m, i),(y, n, j))= \begin{cases}0, & \text { if } i=j \\ \frac{1-\xi_{j}}{1-\xi_{i}} \sum_{s \in \mathscr{P}(y)}\left[k_{i}^{(n-1)}(o, s) p(s, y)\right], & \text { if } i \neq j\end{cases}
$$


For convenience, we write $q((x, m, i),(y, n, j)):=0$, if $j \in \mathscr{I}, y \in V_{j}$, but $(y, n, j) \notin \mathscr{A}$. As the probabilities $q((x, m, i),(y, n, j))$ do not depend on $x$ and $m$, the sequence $\left(\tau_{k}\right)_{k \in \mathbb{N}}$ is also a Markov chain on the state space $\mathscr{I}$ with transition probabilities

$$
\hat{q}(i, j):=\sum_{y \in V_{j}^{\times}} \sum_{n \geq 1} q((x, m, i),(y, n, j))
$$

for $i, j \in \mathscr{I}$ with $i \neq j$ and $\hat{q}(i, i)=0$. Note that $x \in V_{i}$ and $m \in \mathbb{N}$ can be chosen arbitrarily, such that $(x, m, i) \in \mathscr{A}$. As $\mathscr{I}$ is finite, $\left(\tau_{k}\right)_{k \in \mathbb{N}}$ possesses an invariant probability measure $v: \mathscr{I} \rightarrow[0,1]$, that is, for every $j \in \mathscr{I}$

$$
\sum_{i \in \mathscr{I}} v(i) \hat{q}(i, j)=v(j) \text {. }
$$

We now define for $j \in \mathscr{I}, y \in V_{j}^{\times}$and $n \in \mathbb{N}$

$$
\pi(y, n, j):=\sum_{i \in \mathscr{I}} \nu(i) q((x, m, i),(y, n, j))
$$

which is an invariant probability measure of the stochastic process $\left(\widetilde{W}_{k}, \mathbf{i}_{k}, \tau_{k}\right)_{k \in \mathbb{N}}$, that is,

$$
\sum_{(x, m, i) \in \mathscr{A}} \pi(x, m, i) q((x, m, i),(y, n, j))=\pi(y, n, j)
$$

holds for all $(y, n, j) \in \mathscr{A}$.

PROPOSITION 3.2. There is a number $\Lambda \in \mathbb{R}$, such that $\mathbf{e}_{k} / k \stackrel{k \rightarrow \infty}{\longrightarrow} \Lambda \mathbb{P}_{o}$-a.s.

PROOF. Consider the function $g: \mathscr{A} \rightarrow \mathbb{N},(y, n, j) \mapsto n$. An application of the ergodic theorem for positive recurrent Markov chains shows that

$$
\frac{1}{k} \sum_{l=1}^{k} g\left(\tilde{W}_{l}, \mathbf{i}_{l}, \tau_{l}\right)=\frac{\mathbf{e}_{k}-\mathbf{e}_{0}}{k}=\frac{\mathbf{e}_{k}}{k} \stackrel{k \rightarrow \infty}{\longrightarrow} \int g d \pi \quad \mathbb{P}_{o} \text {-a.s. }
$$

if $\int g d \pi<\infty$ holds. Hence it is sufficient to show finiteness of this integral. Noting that

$$
\begin{aligned}
\int g d \pi & =\sum_{i \in \mathscr{I}} v(i) \sum_{(y, n, j) \in \mathscr{\infty}} n q((x, m, i),(y, n, j)) \\
& =\sum_{i \in \mathscr{I}} \frac{v(i)}{1-\xi_{i}} \sum_{j \in \mathscr{T} \backslash\{i\}} \alpha_{j}\left(1-\xi_{j}\right) \underbrace{\sum_{n \geq 1} n \sum_{y \in V_{j}^{x}} \sum_{s \in \mathscr{P}(y)} k_{i}^{(n-1)}(o, s) p_{j}(s, y),}_{(*)}
\end{aligned}
$$


we now interpret the sum $(*)$ as a power series evaluated at 1 . We have

$$
\begin{aligned}
\sum_{n \geq 1} n & \sum_{y \in V_{j}^{\times}} \sum_{s \in \mathscr{P}(y)} k_{i}^{(n-1)}(o, s) p_{j}(s, y) z^{n-1} \\
=\frac{\partial}{\partial z} & {[\underbrace{\sum_{n \geq 1} \sum_{y \in V_{j}^{x}} \sum_{s \in \mathscr{P}(y)} k_{i}^{(n-1)}(o, s) p_{j}(s, y) z^{n}}_{=: y_{i, j}(z)}] . }
\end{aligned}
$$

It is sufficient to show that the sum $\gamma_{i, j}(z)$ has radius of convergence $R_{i, j}>1$ for all $i, j \in \mathscr{I}$ with $i \neq j$.

$$
\begin{aligned}
\gamma_{i, j}(z) & =\sum_{n \geq 1} \sum_{y \in V_{j}^{\times}} \sum_{s \in \mathscr{P}(y)} k_{i}^{(n-1)}(o, s) p_{j}(s, y) z^{n} \\
& =\sum_{n \geq 1} \sum_{s \in V_{j}} k_{i}^{(n-1)}(o, s) z^{n}-\sum_{n \geq 1} \sum_{s \in \mathscr{P}\left(o_{j}\right)} k_{i}^{(n-1)}(o, s) p_{j}\left(s, o_{j}\right) z^{n} \\
& =z \underbrace{\sum_{y \in V_{j}} K_{i}(o, y \mid z)}_{(* *)}-z \underbrace{\sum_{s \in \mathscr{P}\left(o_{j}\right)} K_{i}(o, s \mid z) p_{j}\left(s, o_{j}\right)}_{(* * *)} .
\end{aligned}
$$

From equation (3.1) we obtain

$$
\sum_{y \in V_{j}} K_{i}(o, y \mid z)=\sum_{y \in V_{j}} \frac{L(o, y \mid z)}{1-\bar{H}_{i}(z)}=\frac{1}{1-\bar{H}_{i}(z)} \sum_{y \in V_{j}} L_{j}\left(o_{j}, y \mid \xi_{j}(z)\right),
$$

and also

$$
\begin{aligned}
\sum_{y \in V_{j}} L_{j}\left(o_{j}, y \mid \xi_{j}(z)\right) & =\frac{1}{G_{j}\left(o_{j}, o_{j} \mid \xi_{j}(z)\right)} \sum_{y \in V_{j}} G_{j}\left(o_{j}, y \mid \xi_{j}(z)\right) \\
& =\frac{1}{G_{j}\left(o_{j}, o_{j} \mid \xi_{j}(z)\right)} \frac{1}{1-\xi_{j}(z)} .
\end{aligned}
$$

Thus the sum $(* *)$ has radius of convergence greater than 1. Furthermore, by (3.1) and Lemmas 2.1 and 2.2,

$$
\begin{aligned}
\sum_{\left.s \in \mathscr{P}_{(o j}\right)} & p_{j}\left(s, o_{j}\right) K_{i}(o, s \mid z) \\
= & \frac{1}{1-\bar{H}_{i}(z)} \sum_{s \in \mathscr{P}_{\left(o_{j}\right)}} p_{j}\left(s, o_{j}\right) \frac{G_{j}\left(o_{j}, s \mid \xi_{j}(z)\right)}{G_{j}\left(o_{j}, o_{j} \mid \xi_{j}(z)\right)} \\
= & \frac{1}{\left(1-\bar{H}_{i}(z)\right) G_{j}\left(o_{j}, o_{j} \mid \xi_{j}(z)\right)} \sum_{s \in \mathscr{P}_{(o j)}} p_{j}\left(s, o_{j}\right) \xi_{j}(z) G_{j}\left(o_{j}, s \mid \xi_{j}(z)\right) \frac{1}{\xi_{j}(z)} \\
= & \frac{G_{j}\left(o_{j}, o_{j} \mid \xi_{j}(z)\right)-1}{\left(1-\bar{H}_{i}(z)\right) G_{j}\left(o_{j}, o_{j} \mid \xi_{j}(z)\right) \xi_{j}(z)} .
\end{aligned}
$$


Thus the sum (***) has radius of convergence greater than 1 , and so does $\gamma_{i, j}(z)$, whence $\int g d \pi$ is finite.

Using the above, we can rewrite $\gamma_{i, j}(z)$ as

$$
\gamma_{i, j}(z)=\frac{1}{\alpha_{i}} \frac{\xi_{i}(z)}{\xi_{j}(z)}\left(\frac{1}{\left(1-\xi_{j}(z)\right) G_{j}\left(o_{j}, o_{j} \mid \xi_{j}(z)\right)}-1\right)
$$

and therefore

$$
\Lambda=\sum_{\substack{i, j \in \mathscr{J} \\ i \neq j}} v(i) \alpha_{j} \frac{1-\xi_{j}}{1-\xi_{i}} \gamma_{i, j}^{\prime}(1) .
$$

The following theorem is now obtained precisely as in Nagnibeda and Woess [16, proof of Theorem D].

THEOREM 3.3. $\frac{\ell\left(Z_{n}\right)}{n} \quad \stackrel{n \rightarrow \infty}{\longrightarrow} \quad \ell=\frac{1}{\Lambda} \quad \mathbb{P}_{o}$-a.s.

Finally, we show how to compute the invariant probability measure $v$ explicitly. For this purpose, it is sufficient to compute the transition probabilities $\hat{q}(i, j)$ for all $i, j \in \mathscr{I}$. By solving the system of linear equations given by equation (3.3), $v$ is obtained. The next lemma proposes a formula for $\hat{q}(i, j)$.

Lemma 3.4. Let $i, j \in \mathscr{I}$ with $i \neq j$. Then $\hat{q}(i, i)=0$ and $\hat{q}(i, j)=\frac{\alpha_{j}}{\alpha_{i}} \frac{\xi_{i}}{\xi_{j}} \frac{1-\xi_{j}}{1-\xi_{i}}\left(\frac{1}{\left(1-\xi_{j}\right) G_{j}\left(\xi_{j}\right)}-1\right)$, where $G_{j}\left(\xi_{j}\right):=G_{j}\left(o_{j}, o_{j} \mid \xi_{j}\right)$.

Proof. By definition of $W_{k}$ and $\mathbb{P}_{o}\left[Z_{\infty} \in V_{\infty}\right]=1$, it follows that $\hat{q}(i, i)=0$. Considering (3.2) and the computations in the proof of Proposition 3.2 leads to $\hat{q}(i, j)=\left(1-\xi_{j}\right) /\left(1-\xi_{i}\right) \cdot \alpha_{j} \cdot \gamma_{i, j}(1)$.

We now give an explicit formula for $\nu$.

$$
\nu(i)=c \frac{\alpha_{i}\left(1-\xi_{i}\right)}{\xi_{i}}\left(1-\left(1-\xi_{i}\right) G_{i}\left(\xi_{i}\right)\right)
$$

where $c>0$ is chosen so that $\sum_{i \in \mathscr{I}} v(i)=1$. This is indeed an invariant measure, because, writing $x(i)=1-\left(1-\xi_{i}\right) G_{i}\left(\xi_{i}\right)$, the invariance condition on $v$ is just

$$
\sum_{i \in \mathscr{I} \backslash\langle j\}} x(i)=\frac{x(j)}{\frac{1}{\left(1-\xi_{j}\right) G_{j}\left(\xi_{j}\right)}-1} \text { for each } j \in \mathscr{I}
$$

or, equivalently, that $\sum_{i \in \mathscr{I}} x(i)=1$.

The following lemma verifies that this equation holds. 
LEMMA 3.5. Let $i \in \mathscr{I}$. Then

$$
\rho(i):=\mathbb{P}_{o}\left[Z_{\infty}^{(1)} \in V_{i}^{\times}, \forall n \in \mathbb{N}: Z_{n} \notin \bigcup_{j \in \mathscr{T} \backslash\{i\}} V_{j}\right]=\frac{1-\left(1-\xi_{i}\right) G_{i}\left(\xi_{i}\right)}{G(o, o \mid 1)}
$$

Proof. By transience, $o$ is visited only finitely often $\mathbb{P}_{e}$-a.s., that is,

$$
\sum_{i \in \mathscr{I}} G(o, o \mid 1) \rho(i)=1 \text {. }
$$

This yields

$$
\begin{aligned}
\rho(i) & =\sum_{y \in V_{i}^{\times}} \sum_{j \in \mathscr{I} \backslash(i)} L(o, y \mid 1) \rho(j) \\
& =\sum_{y \in V_{i}^{\times}} L(o, y \mid 1)\left(G(o, o \mid 1)^{-1}-\rho(i)\right) \\
& =\left(G(o, o \mid 1)^{-1}-\rho(i)\right) \sum_{y \in V_{i}^{\mathrm{x}}} \frac{G_{i}\left(o_{i}, y \mid \xi_{i}\right)}{G_{i}\left(\xi_{i}\right)} \\
& =\left(G(o, o \mid 1)^{-1}-\rho(i)\right)\left(\frac{1}{\left(1-\xi_{i}\right) G_{i}\left(\xi_{i}\right)}-1\right) .
\end{aligned}
$$

This leads to the proposed equation.

We summarize this section. If we know for each factor $V_{i}, i \in \mathscr{I}$, the first visit generating function $F_{i}\left(x, o_{i} \mid z\right)$, when starting at a predecessor $x \in \mathscr{P}\left(o_{i}\right)$ of $o_{i}$, and the Green function $G_{i}\left(o_{i}, o_{i} \mid z\right)$, it is possible to compute $\xi_{i}(z)$ by solving a finite system of characteristic equations. This is in fact only possible when the generating functions are known and not too complicated. The measure $v$ can be computed by (3.6) and then $\ell$ found using $\ell=1 / \Lambda$ and (3.5). Sample computations are presented in Section 6.

\section{Double generating functions}

In this section we compute the rate of escape $\ell$ for the random walk on $V$ using double generating functions. The main tool for our computation is the following theorem.

THEOREM 4.1 (Sawyer and Steger). Let $\left(Y_{n}\right)_{n \in \mathbb{N}_{0}}$ be a sequence of real-valued random variables such that, for some $\delta>0$,

$$
\mathbb{E}\left(\sum_{n \geq 0} \exp \left(-r Y_{n}-s n\right)\right)=\frac{C(r, s)}{g(r, s)} \text { for } 0<r, s<\delta,
$$


where $C(r, s)$ and $g(r, s)$ are analytic for $|r|,|s|<\delta$ and $C(0,0) \neq 0$. Denote by $g_{r}$ and $g_{s}$ the partial derivatives of $g$ with respect to $r$ and $s$. Then

$$
\frac{Y_{n}}{n} \stackrel{n \rightarrow \infty}{\longrightarrow} \ell=\frac{g_{r}(0,0)}{g_{s}(0,0)} \quad \text { almost surely. }
$$

For the proof, see Sawyer and Steger [17, Theorem 2.2].

Setting $Y_{n}=\ell\left(Z_{n}\right), w=e^{-r}$ and $z=e^{-s}$, to find $\ell$ in our context it is sufficient to investigate the double generating function

$$
\mathscr{E}(w, z):=\sum_{x \in V} \sum_{n \geq 0} p^{(n)}(o, x) w^{\ell(x)} z^{n}=\sum_{x \in V} G(o, x \mid z) w^{\ell(x)}
$$

and to apply Theorem 4.1. To this end, introduce further double generating functions. Write $V^{\times}:=V \backslash\{o\}$ and define

$$
\mathscr{L}(w, z):=1+\sum_{n \geq 1} \sum_{x=x_{1} \cdots x_{n} \in V^{\times}} \prod_{j=1}^{n} w L_{\tau\left(x_{j}\right)}\left(o_{\tau\left(x_{j}\right)}, x_{j} \mid \xi_{\tau\left(x_{j}\right)}(z)\right)
$$

and for $i \in \mathscr{I}$

$$
\begin{aligned}
\mathscr{L}_{i}^{+}(w, z) & :=\sum_{x \in V_{i}^{\mathrm{x}}} L_{i}\left(o_{i}, x \mid \xi_{i}(z)\right) w, \\
\mathscr{L}_{i}(w, z) & :=\mathscr{L}_{i}^{+}(w, z)\left(1+\sum_{\substack { n \geq 2 \\
\begin{subarray}{c}{x_{2}, \ldots, x_{n} \in V^{\mathrm{x}} \\
\tau\left(x_{2}\right) \neq i{ n \geq 2 \\
\begin{subarray} { c } { x _ { 2 } , \ldots , x _ { n } \in V ^ { \mathrm { x } } \\
\tau ( x _ { 2 } ) \neq i } }\end{subarray}} \prod_{j=2}^{n} w L_{\tau\left(x_{j}\right)}\left(o_{\tau\left(x_{j}\right)}, x_{j} \mid \xi_{\tau\left(x_{j}\right)}(z)\right)\right) .
\end{aligned}
$$

Thus we have the equation

$$
\mathscr{L}(w, z)=1+\sum_{i \in \mathscr{I}} \mathscr{L}_{i}(w, z)
$$

If $0 \leq w, z<1$, the convergence of $\mathscr{L}(w, z)$ follows by $\mathscr{L}(w, z) \leq \mathscr{E}(w, z)$. Hence, $\mathscr{L}_{i}^{+}(w, z)$ and also $\mathscr{L}_{i}(w, z), i \in \mathscr{I}$, converge if $|w|,|z|<1$. The next lemma provides another representation of $\mathscr{L}(w, z)$.

LEMMA 4.2. Let $w, z \in \mathbb{R}$ with $0 \leq w, z<1$. Then

$$
\mathscr{L}(w, z)=\frac{1}{1-\mathscr{L}^{*}(w, z)}, \quad \text { where } \quad \mathscr{L}^{*}(w, z)=\sum_{i \in \mathscr{I}} \frac{\mathscr{L}_{i}^{+}(w, z)}{1+\mathscr{L}_{i}^{+}(w, z)}
$$

PROOF. Let $w, z \in \mathbb{R}$ with $0<w, z<1$. First we have

$$
\mathscr{L}_{i}(w, z)=\mathscr{L}_{i}^{+}(w, z)\left(1+\sum_{j \in \mathscr{I} \backslash i i\}} \mathscr{L}_{j}(w, z)\right) \quad \text { for all } i \in \mathscr{I},
$$


and from convergence of $\mathscr{L}(w, z)$, we get

$$
\mathscr{L}_{i}(w, z)=\mathscr{L}_{i}^{+}(w, z)\left(\mathscr{L}(w, z)-\mathscr{L}_{i}(w, z)\right) .
$$

As $\mathscr{L}_{i}^{+}(w, z) \geq 0$ holds, the last equation is equivalent to

$$
\mathscr{L}_{i}(w, z)=\frac{\mathscr{L}_{i}^{+}(w, z)}{1+\mathscr{L}_{i}^{+}(w, z)} \mathscr{L}(w, z)
$$

Thus

$$
\mathscr{L}(w, z)=1+\sum_{i \in \mathscr{I}} \mathscr{L}_{i}(w, z)=1+\sum_{i \in \mathscr{I}} \frac{\mathscr{L}_{i}^{+}(w, z)}{1+\mathscr{L}_{i}^{+}(w, z)} \mathscr{L}(w, z)
$$

As $\mathscr{L}(w, z)<\infty$, we get

$$
\sum_{i \in \mathscr{I}} \frac{\mathscr{L}_{i}^{+}(w, z)}{1+\mathscr{L}_{i}^{+}(w, z)}<1
$$

and the result follows.

COROllary 4.3. Let $w, z \in \mathbb{R}$ with $0 \leq w, z<1$. Then

$$
\mathscr{E}(w, z)=\frac{G(o, o \mid z)}{1-\mathscr{L}^{*}(w, z)} .
$$

ProOF. Let $w, z \in \mathbb{R}$ with $0<w, z<1$. Applying Lemma 4.2 yields the proposed equation:

$$
\begin{aligned}
\mathscr{E}(w, z) & =\sum_{x \in V} G(o, o \mid z) L(o, x \mid z) w^{\ell(x)} \\
& =G(o, o \mid z)\left(1+\sum_{n \geq 1} \sum_{x=x_{1} \cdots x_{n} \in V^{x}} L(o, x \mid z) w^{\ell(x)}\right) \\
& =G(o, o \mid z) \mathscr{L}(w, z) .
\end{aligned}
$$

We can now conclude and compute a formula for the rate of escape $\ell$. Rewriting $\mathscr{L}^{*}(w, z)$ after some manipulations involving Lemma 2.1 yields

$$
\mathscr{L}^{*}(w, z)=\sum_{i \in \mathscr{I}} \frac{w\left(1 /\left(1-\xi_{i}(z)\right)-G_{i}\left(o_{i}, o_{i} \mid \xi_{i}(z)\right)\right)}{w /\left(1-\xi_{i}(z)\right)+(1-w) G_{i}\left(o_{i}, o_{i} \mid \xi_{i}(z)\right)} .
$$

We define $C(w, z):=G(o, o \mid z)$ and

$$
g(w, z):=1-\sum_{i \in \mathscr{I}} \frac{w\left(1 /\left(1-\xi_{i}(z)\right)-G_{i}\left(o_{i}, o_{i} \mid \xi_{i}(z)\right)\right)}{w /\left(1-\xi_{i}(z)\right)+(1-w) G_{i}\left(o_{i}, o_{i} \mid \xi_{i}(z)\right)} .
$$


We have

$$
\mathscr{E}(w, z)=\frac{C(w, z)}{g(w, z)} \quad \text { for } 0 \leq w, z<1
$$

The constraints required for an application of Theorem 4.1 are obviously fulfilled, as $G(o, o \mid z)$ has radius of convergence greater than 1 and $\xi_{i}<1$. We apply it now, where $g_{w}$ and $g_{z}$ denote the partial derivatives of $g$ with respect to $w$ and $z$, respectively. Hence, we can conclude

$$
\frac{\ell\left(Z_{n}\right)}{n} \stackrel{n \rightarrow \infty}{\longrightarrow} \ell=\frac{g_{w}(1,1)}{g_{z}(1,1)} \quad \mathbb{P}_{o} \text {-a.s. }
$$

Simplifications yield the following formula for $\ell$, where we write $\xi_{i}=\xi_{i}(1)$ and $G_{i}\left(\xi_{i}\right):=G_{i}\left(o_{i}, o_{i} \mid \xi_{i}\right)$.

$$
\ell=\frac{\sum_{i \in \mathscr{I}}\left[\left(1-\left(1-\xi_{i}\right) G_{i}\left(\xi_{i}\right)\right) \cdot G_{i}\left(\xi_{i}\right) \cdot\left(1-\xi_{i}\right)\right]}{\sum_{i \in \mathscr{I}}\left[\xi_{i}^{\prime}(1) \cdot\left(G_{i}\left(\xi_{i}\right)-\left(1-\xi_{i}\right) G_{i}^{\prime}\left(\xi_{i}\right)\right)\right]} .
$$

Observe that $\xi_{i}, G_{i}\left(\xi_{i}\right)>0, \xi_{i}<1$ and $G_{i}\left(\xi_{i}\right)<\left(1-\xi_{i}\right)^{-1}$. Thus $\ell>0$.

\section{Free products of groups}

In this section we present a third technique for the computation of the rate of escape $\ell$ of the block length for the random walk on the free product. This technique is restricted to the case of a free product of groups. Therefore, let $\Gamma_{i}, i \in \mathscr{I}$, be non-trivial finitely generated groups. We assume that the groups have pairwise trivial intersections, but they may be isomorphic. Denote by $e_{i}$ the identity on $\Gamma_{i}$. The elements of the free product $\Gamma:=\Gamma_{1} * \cdots * \Gamma_{r}$ are represented as words in the sense of (2.1) and $e$ is identified with the empty word.

We can define a group operation on $\Gamma$. The product of $u, v \in \Gamma$ is the concatenation of the words $u$ and $v$ with possible cancellations and contractions in the middle to get the representative form of the product word. We exclude the case $r=2=\left|\Gamma_{1}\right|=\left|\Gamma_{2}\right|$. This ensures that the free group product is non-amenable, yielding that each of our constructed random walks on $\Gamma$ is transient and $G(e, e \mid z)$ has radius of convergen'ce greater than 1 (see Woess [23, Theorem 10.10, Proposition 12.4, Corollary 12.5]). We write $\Gamma_{i}^{\times}=\Gamma_{i} \backslash\left\{e_{i}\right\}$ for $i \in \mathscr{I}$. Furthermore we write $\Gamma_{\infty}=V_{\infty}$ and $\Gamma^{\times}:=\Gamma \backslash\{e\}$.

The random walk on $\Gamma$ is constructed as follows: standing at $x \in \Gamma$ we allow walking to $x g$ with $g \in \bigcup_{i \in \mathcal{I}} \Gamma_{i}^{\times}$in one step. Choose probability measures $\mu_{i}$ on $\Gamma_{i}^{\times}$for each $i \in \mathscr{I}$ such that $\mu_{i}$ defines an irreducible random walk on $\Gamma_{i}$, that is, 
$p_{i}(x, y)=\mu_{i}\left(x^{-1} y\right)$ for all $x, y \in \Gamma_{i}$. Let $\alpha_{1}, \ldots, \alpha_{r}>0$ with $\sum_{i \in \mathscr{I}} \alpha_{i}=1$. Then we define the transition probabilities as

$$
p(x, x g)=\alpha_{i} \cdot \mu_{i}(g)
$$

for all $x \in \Gamma, g \in \Gamma_{i}^{\mathrm{x}}$, and we set $p(x, y)=0$ otherwise. As the transition probabilities depend only on the increment $g \in \Gamma_{i}^{\times}$, we write $\mu(g):=p(x, x g)$ for all $g \in \bigcup_{i \in \mathscr{I}} \Gamma_{i}^{\times}$, and $\mu(g)=0$ otherwise. Analogously, the n-step transition probabilities are given by the convolution powers $\mu^{(n)}$ of $\mu$.

By Theorem 2.5, the random walk converges $\mathbb{P}_{e}$-a.s. to a random variable $Z_{\infty}$ with values in $\Gamma_{\infty}$. Denote by $\nu$ the distribution of $Z_{\infty}$. Let

$$
E_{i}=\left\{x_{1} x_{2} \cdots \in \Gamma_{\infty} \mid \tau\left(x_{1}\right)=i\right\} \quad \text { for } i \in \mathscr{I} .
$$

Then $v$ is uniquely determined by its values on the Borel sets $B$ of the form $x E_{i}=$ $\left\{x h \mid h \in E_{i}\right\}$ with $i \in \mathscr{I}, x \in \Gamma$ and $\tau(x) \neq i$. We will now give a formula for these values.

LeMMA 5.1. Let $i \in \mathscr{I}, x \in \Gamma$ with $\tau(x) \neq i$. Then

$$
\nu\left(x E_{i}\right):=\mathbb{P}_{e}\left[Z_{\infty} \in x E_{i}\right]=F(e, x \mid 1)\left(1-\left(1-\xi_{i}\right) G_{i}\left(e_{i}, e_{i} \mid \xi_{i}\right)\right) .
$$

Proof. The proof of this lemma is extrapolated from Woess [21, Theorem $4 \mathrm{c}$ ], where one can find an incorrect formula, which we correct here. First we have

$$
\nu\left(x E_{i}\right)=F(e, x \mid 1) \cdot \nu\left(E_{i}\right) .
$$

Recall that we have by vertex-transitivity $G_{i}\left(o_{i}, o_{i} \mid z\right)=G_{i}(y, y \mid z)$ for all $i \in \mathscr{I}$ and all $y \in \Gamma_{i}$. By Lemma 3.5, we obtain

$$
\nu\left(E_{i}\right)=G(o, o \mid 1) \cdot \rho(i)=1-\left(1-\xi_{i}\right) G_{i}\left(e_{i}, e_{i} \mid \xi_{i}\right) .
$$

This leads to the proposed formula.

Now we reformulate our problem for finding a formula for $\ell$. For this purpose, we apply a technique which was used by Ledrappier [10, Section $4 \mathrm{~b}$ ] for free groups.

By Theorem 3.3 and Lebesgue's Dominated Convergence Theorem, we have $\mathbb{P}_{e}$-a.s.

$$
\lim _{n \rightarrow \infty} \frac{\mathbb{E}\left[\ell\left(Z_{n}\right)\right]}{n}=\lim _{n \rightarrow \infty} \int \frac{\ell\left(Z_{n}\right)}{n} d \mathbb{P}_{e}=\int \lim _{n \rightarrow \infty} \frac{\ell\left(Z_{n}\right)}{n} d \mathbb{P}_{e}=\int \ell d \mathbb{P}_{e}=\ell .
$$

Thus it is sufficient to prove convergence of the sequence $\left(\mathbb{E}\left[\ell\left(Z_{n+1}\right)\right]-\mathbb{E}\left[\ell\left(Z_{n}\right)\right]\right)_{n \in \mathbb{N}}$ and to compute its limit, which then must equal $\ell$. First we have

$$
\mathbb{E}\left[\ell\left(Z_{n}\right)\right]=\sum_{h \in \Gamma} \ell(h) \mu^{(n)}(h) \quad \text { and } \quad \mathbb{E}\left[\ell\left(Z_{n+1}\right)\right]=\sum_{g, h \in \Gamma} \ell(g h) \mu(g) \mu^{(n)}(h) .
$$


On the other hand,

$$
\mathbb{E}\left[\ell\left(Z_{n}\right)\right]=\sum_{g \in \Gamma} \mu(g) \mathbb{E}\left[\ell\left(Z_{n}\right)\right]=\sum_{g, h \in \Gamma} \mu(g) \ell(h) \mu^{(n)}(h) .
$$

Thus we obtain

$$
\begin{aligned}
\mathbb{E}\left[\ell\left(Z_{n+1}\right)\right]-\mathbb{E}\left[\ell\left(Z_{n}\right)\right] & =\sum_{g \in \Gamma} \mu(g) \sum_{h \in \Gamma}(\ell(g h)-\ell(h)) \mu^{(n)}(h) \\
& =\sum_{g \in \Gamma} \mu(g) \int\left(\ell\left(g Z_{n}\right)-\ell\left(Z_{n}\right)\right) d \mathbb{P}_{e}
\end{aligned}
$$

Define the random variables $Y_{n}:=\ell\left(g Z_{n}\right)-\ell\left(Z_{n}\right)$ for any given $g \in \bigcup_{i \in \mathscr{I}} \Gamma_{i}^{\times}$. We have $Y_{n} \in\{-1,0,1\}$ for all $n \in \mathbb{N}$. By vertex-transitivity, $g Z_{n}$ converges to $g Z_{\infty}$. Hence, $Y_{n}$ converges to a random variable $Y_{\infty}$ with values in $\{-1,0,1\}$ depending only on $g$ and the first block of $Z_{\infty}$. In other words, $Y_{n}$ becomes constant, if $n$ is big enough. If $Z_{\infty}=x_{1} x_{2} \cdots$, we obtain for a given $g \in \bigcup_{i \in \mathscr{I}} \Gamma_{i}^{\times}$

$$
Y_{\infty}= \begin{cases}0, & \text { if } \tau\left(x_{1}\right)=\tau(g) \text { and } x_{1} g \neq e \\ -1, & \text { if } \tau\left(x_{1}\right)=\tau(g) \text { and } x_{1} g=e \\ 1, & \text { if } \tau\left(x_{1}\right) \neq \tau(g)\end{cases}
$$

By Lebesgue's Dominated Convergence Theorem, we infer that

$$
\int_{\Gamma}\left(\ell\left(g Z_{n}\right)-\ell\left(Z_{n}\right)\right) d \mathbb{P}_{e} \stackrel{n \rightarrow \infty}{\longrightarrow} \int Y_{\infty} d \mathbb{P}_{e}
$$

Consider the function

$$
f:\left(\bigcup_{i \in \mathscr{I}} \Gamma_{i}^{\times}\right) \times \Gamma_{\infty} \rightarrow\{-1,0,1\}:\left(g, x_{1} x_{2} \cdots\right) \mapsto \ell\left(g x_{1}\right)-\ell\left(x_{1}\right)
$$

and its projections $f_{g}: \dot{\Gamma}_{\infty} \rightarrow\{-1,0,1\}: w \mapsto f(g, w)$ for every $g \in \bigcup_{i \in \mathscr{I}} \Gamma_{i}^{\times}$. Each $f_{g}$ is measurable and thus

$$
\int Y_{\infty} d \mathbb{P}_{e}=\int f\left(g, Z_{\infty}\right) d v=\int_{\Gamma^{\infty}} f_{g}(w) d v(w)
$$

Denote by $E_{h}$ the event that $Z_{\infty}$ has as first block the element $h \in \bigcup_{i \in \mathscr{I}} \Gamma_{i}^{\times}$and denote $E_{\neq i}$ the event that $Z_{\infty}$ starts with a block element not of type $i \in \mathscr{I}$. Then we obtain for $g \in \Gamma_{i}$

$$
\nu\left(E_{g^{-1}}\right)=F\left(e, g^{-1} \mid 1\right) \cdot\left(1-\nu\left(E_{i}\right)\right)=F\left(e, g^{-1} \mid 1\right) \cdot\left(1-\xi_{i}\right) \cdot G_{i}\left(e_{i}, e_{i} \mid \xi_{i}\right)
$$


and $\left.\nu\left(E_{\neq i}\right)=\left(1-\xi_{i}\right) \cdot G_{i}\left(e_{i}, e_{i} \mid \xi_{i}\right)\right)$. Writing $G_{i}(z)$ for $G_{i}\left(e_{i}, e_{i} \mid z\right)$, as before, we can conclude

$$
\begin{aligned}
\mathbb{E}\left[\ell\left(Z_{n+1}\right)\right]-\mathbb{E}\left[\ell\left(Z_{n}\right)\right] & \stackrel{n \rightarrow \infty}{\longrightarrow} \sum_{g \in \bigcup_{i \in \mathscr{S}} \Gamma_{i}^{\times}} \mu(g) \int_{\Gamma^{\infty}} f_{g}(w) d v(w) \\
& =\sum_{g \in \cup_{i \in \mathscr{S}} \Gamma_{i}^{\times}} \mu(g)\left(-v\left(E_{g^{-1}}\right)+v\left(E_{\neq i}\right)\right) . \\
& =\sum_{i \in \mathscr{I}} \alpha_{i}\left(1-\xi_{i}\right) G_{i}\left(\xi_{i}\right)(1-\underbrace{\left.\sum_{g \in \Gamma_{i}^{\times}} \mu_{i}(g) F_{i}\left(e_{i}, g^{-1} \mid \xi_{i}\right)\right)}_{=\left(G_{i}\left(\xi_{i}\right)-1\right) /\left(\xi_{i} \cdot G_{i}\left(\xi_{i}\right)\right)} \\
& =\sum_{i \in \mathscr{I}} \alpha_{i} \frac{1-\xi_{i}}{\xi_{i}}\left(1-\left(1-\xi_{i}\right) G_{i}\left(\xi_{i}\right)\right) .
\end{aligned}
$$

Thus we get the rate of escape of the block length

$$
\ell=\sum_{i \in \mathscr{I}} \alpha_{i} \frac{1-\xi_{i}}{\xi_{i}}\left(1-\left(1-\xi_{i}\right) G_{i}\left(\xi_{i}\right)\right) .
$$

The technique presented in this section can be extended to a free product of an infinite, countable number of groups. All the required properties of the generating functions used also hold in this case. Furthermore, $Y_{n}$ is again bounded such that finiteness of $\ell$ is ensured. Thus the same computations prove the same formula for $\ell$ if $\mathscr{I}=\mathbb{N}$.

\section{Examples}

We present two applications of our formulae for the rate of escape of the block length. First we look at a free product arising from non-Cayley graphs and then we look at a free product of infinite groups. Both examples go beyond previously investigated graph structures for the computation of $\ell$.

\subsection{Free product arising from non-Cayley-graphs Consider the sets}

$$
V_{1}=\left\{A, B, C, D, E, F, o_{1}\right\}, \quad V_{2}=\left\{G, H, o_{2}\right\} \quad \text { and } \quad V_{3}=\left\{I, J, o_{3}\right\},
$$

and the random walks on these sets. Their transition probabilities are sketched in Figure 1. None of the graphs in this figure is a Cayley graph.

Consider now the corresponding random walk on the free product $V=V_{1} * V_{2} * V_{3}$, where $\alpha_{1}=5 / 9$ and $\alpha_{2}=\alpha_{3}=2 / 9$.

We obtain the generating functions

$$
U_{1}\left(o_{1}, o_{1} \mid z\right)=\frac{3}{5} z^{2}+\frac{2}{5} z^{3}, \quad G_{1}\left(o_{1}, o_{1} \mid z\right)=\frac{1}{1-U_{1}\left(o_{1}, o_{1} \mid z\right)}=\frac{1}{1-\frac{3}{5} z^{2}-\frac{2}{5} z^{3}},
$$



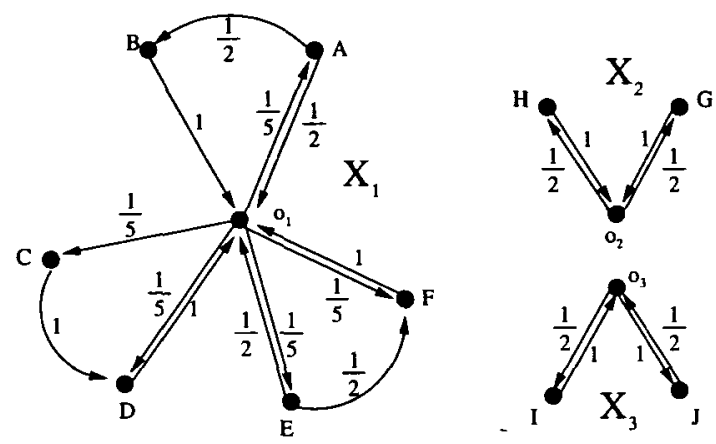

FIGURE 1. Some non-Cayley graphs.

$$
\begin{array}{clrl}
F_{1}\left(A, o_{1} \mid z\right)=F_{1}\left(E, o_{1} \mid z\right)=\frac{1}{2} z+\frac{1}{2} z^{2}, & & F_{1}\left(C, o_{1} \mid z\right)=z^{2}, \\
F_{1}\left(D, o_{1} \mid z\right)=F_{1}\left(F, o_{1} \mid z\right)=z, & G_{2}\left(o_{2}, o_{2} \mid z\right)=\frac{1}{1-z^{2}}, \\
F_{2}\left(G, o_{2}, z\right)=F_{2}\left(H, o_{2} \mid z\right)=z, & \vec{H}_{1}(z)=\frac{4}{9} z \xi_{2}(z), \\
\bar{H}_{2}(z)=\bar{H}_{3}(z)=\frac{2}{9} z \xi_{2}(z)+\frac{1}{9} z\left(3 \xi_{1}(z)+2 \xi_{1}(z)^{2}\right) .
\end{array}
$$

Note that $\bar{H}_{2}(z)=\bar{H}_{3}(z)$ follows by symmetry. The Green function $G(o, o \mid z)$ of the corresponding random walk on $V$ has radius of convergence greater than 1 . This can be shown by constructing recursive equations using $\bar{H}_{1}(z)$ and $\bar{H}_{2}(z)$ and numerical evaluation. Consider

$$
\begin{aligned}
& \xi_{1}(z)=\frac{\frac{5}{9} z}{1-\bar{H}_{1}(z)}=\frac{\frac{5}{9} z}{1-\frac{4}{9} z \xi_{2}(z)} \text { and } \\
& \xi_{2}(z)=\xi_{3}(z)=\frac{\frac{2}{9} z}{1-\bar{H}_{2}(z)}=\frac{\frac{2}{9} z}{1-\frac{2}{9} z \xi_{2}(z)-\frac{1}{9} z\left(3 \xi_{1}(z)+2 \xi_{1}(z)^{2}\right)} .
\end{aligned}
$$

Substituting $\xi_{1}(z)$ into $\xi_{2}(z)$ we have to solve an equation in the variable $\xi_{2}(z)$. Solving this equation with MATHEMATICA we obtain four continuous solutions, but only one solution satisfies $\xi_{2}(1)<1$. Hence, we get $\xi_{2}(z)$ as this solution and obtain $\xi_{1}(z)$ from $\xi_{2}(z)$. We find that $\xi_{1}(1) \approx 0.66571$ and $\xi_{2}(1)=\xi_{3}(1) \approx 0.37231$. We compute $\ell$ using Theorem 3.3. The transition matrix of the Markov chain $\left(\tau_{k}\right)_{k \in \mathbb{N}}$ of the alternating vertex types is

$$
(\hat{q}(i, j))_{1 \leq i, j \leq 3}=\left(\begin{array}{ccc}
0 & 0.5 & 0.5 \\
0.62769 & 0 & 0.37231 \\
0.62769 & 0.37231 & 0
\end{array}\right),
$$


and from this we obtain the corresponding invariant probability measure $v$ with $v(1)=0.38563$ and $v(2)=v(3)=0.30718$. Now we are able to compute the rate of escape of the block length to the random walk on $V$. We obtain $\ell \approx 0.33089$. If $\ell$ is computed by (4.1), then the numerical approximated result and the above result coincide in the first 50 decimal numbers. So numerical approximations do not lead to a distortion of the result.

6.2. $\mathbb{Z}^{2} * \mathbb{Z} / 2$ Consider $\Gamma_{1}=\mathbb{Z}^{2}$ and the simple random walk on it given by $\mu_{1}(( \pm 1,0))=\mu_{1}((0, \pm 1))=1 / 4$. Also, consider the group $\Gamma_{2}=\mathbb{Z} / 2$ and the simple random walk on it given by $\mu_{2}\left(1_{2}\right)=1$. We are interested now in the simple random walk on $\Gamma=\Gamma_{1} * \Gamma_{2}$, where $\alpha_{1}=4 / 5$ and $\alpha_{2}=1 / 5$. For the computation of $\ell$ we use (5.1). Therefore it is sufficient to compute $\xi_{1}, \xi_{2}$ and $G_{1}\left((0,0),(0,0) \mid \xi_{1}\right)$. For this purpose, we use the computations and results in Woess [23, pages 100, 105, 109]; compare also with Soardi [18].

Before we can compute these values, we have to introduce some auxiliary functions. In the following let the subindex 0 correspond to the random walk on $V$. Denote

$$
W_{i}(z)=z \cdot G_{i}(o, o \mid z) \text { for } i \in\{0,1,2\} .
$$

As $W_{i}(z)$ is strictly increasing, there is an inverse function $W_{i}^{-1}(z)$ such that

$$
W_{i}^{-1}\left(W_{i}(z)\right)=z
$$

By [23, Theorem 9.10], we have

$$
G_{i}(o, o \mid z)=\Phi_{i}\left(z G_{i}(o, o \mid z)\right),
$$

where $\Phi_{i}(t)=t / W_{i}^{-1}(t)$ for all $i \in\{0,1,2\}$. By [23, Example 9.15 (3)],

$$
W_{1}(z)=\frac{1}{4 \pi^{2}} \int_{(-\pi, \pi)^{2}} \frac{2 z}{2-z \cdot\left(\cos x_{1}+\cos x_{2}\right)} d \underline{x},
$$

where $\underline{x}=\left(x_{1}, x_{2}\right)$. Furthermore, by [23, Theorem 9.19], we have the equation

$$
\Phi_{0}(t)=\Phi_{1}\left(\alpha_{1} t\right)+\Phi_{2}\left(\alpha_{2} t\right)-1 .
$$

By [23, Example 9.15 (1)], we have

This yields

$$
\Phi_{2}(t)=\frac{1}{2}\left(\sqrt{1+4 t^{2}}+1\right) .
$$

$$
\Phi_{1}\left(\frac{4}{5} W(z)\right)=G(o, o \mid z)-\frac{1}{2}\left(\sqrt{1+\frac{4}{25} W(z)^{2}}-1\right) .
$$


Inverting this equation and multiplying by $(4 / 5) W(z)$ leads to

$$
\frac{\frac{4}{5} W(z)}{\Phi_{1}\left(\frac{4}{5} W(z)\right)}=\frac{\frac{4}{5} W(z)}{G(o, o \mid z)-\frac{1}{2}\left(\sqrt{1+\frac{4}{25} W(z)^{2}}-1\right)} .
$$

Applying $W_{1}$ onto both sides of this equation yields

$$
\frac{4}{5} W(z)=W_{1}\left(\frac{\frac{4}{5} W(z)}{G(o, o \mid z)-\frac{1}{2}\left(\sqrt{1+\frac{4}{25} W(z)^{2}}-1\right)}\right) .
$$

We have

$$
W_{1}\left(\xi_{1}(z)\right)=\frac{4}{5} W(z)
$$

and thus

$$
\xi_{1}(z)=\frac{\frac{4}{5} W(z)}{\frac{W(z)}{z}-\frac{1}{2}\left(\sqrt{1+\frac{4}{25} W(z)^{2}}-1\right)} .
$$

Substituting $y=(4 / 5) W(z)$, we obtain

$$
\xi_{i}=\xi_{1}(1)=\frac{y}{\frac{5 y}{4}-\frac{1}{2}\left(\sqrt{1+\frac{1}{4} y^{2}}-1\right)}
$$

or equivalently $y=\left(4-5 \xi_{1}\right) \xi_{1} /\left(4-10 \xi_{1}+6 \xi_{1}^{2}\right)$. Substituting $y$ into (6.1), we have to solve

$$
\frac{\left(4-5 \xi_{1}\right) \xi_{1}}{4-10 \xi_{1}+6 \xi_{1}^{2}}=W_{1}\left(\xi_{1}\right)
$$

in the unknown variable $\xi_{1}$. The solution can be computed only numerically. Considering the graphs of the functions $(4-5 z) z /\left(4-10 z+6 z^{2}\right)$ and $W_{1}(z)$ we see that there is only one possible intersection point greater than $4 / 5$; see Figure 2 . Using the bisection method, numerical integration and evaluation we obtain $\xi_{1} \approx 0.84426$, and also $W(1)=(5 / 4) y \approx 1.40724$. This yields

$$
G_{1}\left((0,0),(0,0) \mid \xi_{1}\right)=\frac{\frac{4}{5} W(1)}{\xi_{1}(1)} \approx 1.33347 .
$$

By $\xi_{1}=4 /\left(5\left(1-\xi_{2} / 5\right)\right)$, we obtain

$$
\xi_{2} \approx 0.26212, \quad \text { and } \quad G_{2}\left(0,0 \mid \xi_{2}\right)=\frac{1}{1-\xi_{2}^{2}} \approx 1.07378
$$

Now we have computed all necessary characteristical numbers, and the rate of escape of the block length of the simple random walk on $\mathbb{Z}^{2} * \mathbb{Z} / 2$ can be computed by (5.1) as $\ell \approx 0.23386$. 

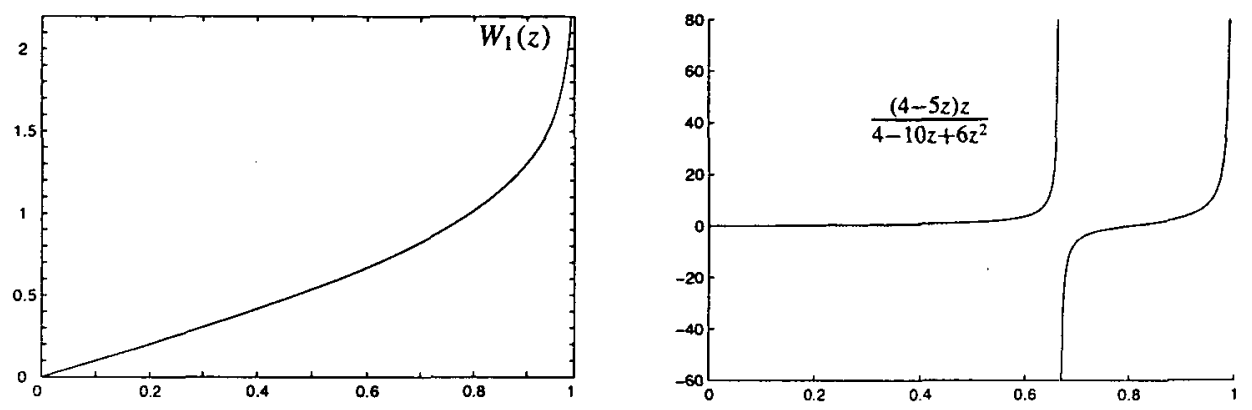

FIGURE 2. Graphs of $W_{1}(z)$ and $(4-5 z) z /\left(4-10 z+6 z^{2}\right)$.

\section{Remarks}

We can extend our considerations in order to compute other types of typical rates of escapes concerning the random walk on the free product:

(I) For $x=x_{1} \cdots x_{n} \in V$ and $i \in \mathscr{I}$ the partial block length of $x$ with respect to $V_{i}$ is given by

$$
\ell_{i}(x):=\left|\left\{j \mid j \in\{1, \ldots, n\}, x_{j} \in V_{i}\right\}\right| .
$$

As $\ell_{i}\left(W_{k}\right) / k$ converges for $k \rightarrow \infty$ to $\nu(i)$, which is the invariant probability measure on $\mathscr{I}$ with respect to the Markov chain $\left(\tau\left(W_{k}\right)\right)_{k \in \mathbb{N}}$, we obtain the partial rate of escape of the block length

$$
\frac{\ell_{i}\left(Z_{n}\right)}{n} \stackrel{n \rightarrow \infty}{\longrightarrow} v(i) \cdot \ell \quad \mathbb{P}_{0} \text {-a.s. }
$$

(II) The set $V$ carries a Markovian distance defined by

$$
d(x, y):=\min \left\{n \in \mathbb{N} \mid p^{(n)}(x, y)>0\right\},
$$

where $x, y \in V$. In general $d(\cdot, \cdot)$ is not necessarily symmetric. The Markovian length is defined as $|x|=d(o, x)$. We can extend the considerations of Section 3 for the proof of the existence of the rate of escape of the Markovian length, that is,

$$
\lambda=\lim _{n \rightarrow \infty} \frac{1}{n}\left|Z_{n}\right| .
$$

This yields a formula for $\lambda$ given by $\lambda=\ell \sigma$, where

$$
\sigma=\sum_{\substack{i, j \in \mathscr{J} \\ i \neq j}} \nu(i) \frac{\alpha_{j}}{\alpha_{i}} \frac{\xi_{i}}{\xi_{j}} \frac{1-\xi_{j}}{1-\xi_{i}} \sum_{m \geq 1} m \sum_{y \in S_{j}(m)} L_{j}\left(o_{j}, y \mid \xi_{j}\right)
$$

and $S_{i}(m)=\left\{x \in V_{i}|| x \mid=m\right\}$ for $i \in \mathscr{I}$ and $m \in \mathbb{N}$. 


\section{Acknowledgement}

The author is grateful to Wolfgang Woess for numerous discussions on several problems and his help during the preparation of this article, and also to Donald Cartwright for several hints regarding content and exposition.

\section{References}

[1] D. I. Cartwright, V. A. Kaimanovich and W. Woess, 'Random walks on the affine group of local fields and of homogeneous trees', Ann. Inst. Fourier (Grenoble) 44 (1994), 1243-1288.

[2] D. I. Cartwright and P. M. Soardi, 'Random walks on free products, quotients, and amalgams', Nagoya Math. J. 102 (1986), 163-180.

[3] Y. Derriennic, 'Quelques applications du théorème ergodique sous-additif', Astérisque 74 (1980), 183-201.

[4] A. Dyubina, 'Characteristics of random walks on wreath products of groups', J. Math. Sci. (5) 107 (2001), 4166-4171.

[5] A. Erschler, 'On the asymptotics of drift', J. Math. Sci. (3) 121 (2004), 2437-2440.

[6] H. Furstenberg, 'Non commuting random products', Trans. Amer. Math. Soc. 108 (1963), 377-428.

[7] Y. Guivarc'h, 'Sur la loi des grands nombres et le rayon spectral d'une marche aléatoire', Astérisque 74 (1980), 47-98.

[8] V. A. Kaimanovich and A. M. Vershik, 'Random walks on discrete groups: boundary and entropy', Ann. Probab. 11 (1983), 457-490.

[9] J. F. C. Kingman, 'The ergodic theory of subadditive processes', J. Roy. Statist. Soc., Ser. B 30 (1968), 499-510.

[10] F. Ledrappier, Some Asymptotic Properties of Random Walks on Free GroupsCRM Proceedings and Lecture Notes 28 (Amer. Math. Soc, Providence, RI, 2001) pp. 117-152.

[11] R. Lyons, R. Pemantle and Y. Peres, 'Random walks on the lamplighter group', Ann. Probab. (4) 24 (1996), 1993-2006.

[12] J. Mairesse, 'Random walks on groups and monoids with a markovian harmonic measure', Technical Report Research Report LIAFA 2004-005, (Univ. Paris 7, 2004).

[13] —_, 'Randomly growing braid on three strands and the manta ray', Technical Report Report LIAFA 2005-001, (Univ. Paris 7, 2005).

[14] J. Mairesse and F. Mathéus, 'Random walks on free products of cyclic groups and on Artin groups with two generators', Technical Report Research Report LIAFA 2004-006, (Univ. Paris 7, 2004).

[15] J. C. McLaughlin, Random walks and convolution operators on free products (Ph.D. Thesis, New York Univ., 1986).

[16] T. Nagnibeda and W. Woess, 'Random walks on trees with finitely many cone types', $J$. Theoret. Probab. 15 (2002), 399-438.

[17] S. Sawyer and T. Steger, 'The rate of escape for anisotropic random walks in a tree', Probab. Theory Related Fields 76 (1987), 207-230.

[18] P. M. Soardi, 'Simple random walks on $\mathbb{Z}^{2} * \mathbb{Z} / 2$ ', Symposia Math. 29 (1986), 303-309.

[19] N. Th. Varopoulos, 'Long range estimates for Markov chains', Bull. Sci. Math. (2) 109 (1985), 225-252.

[20] D. Voiculescu, 'Addition of certain non-commuting random variables', J. Funct. Anal. 66 (1986), 323-346. 
[21] W. Woess, 'A description of the Martin boundary for nearest neighbour random walks on free products', Probability Measures on Groups VII (1985), 203-215.

[22] _-, 'Nearest neighbour random walks on free products of discrete groups', Boll. Un. Mat. Ital. (6) 5-B (1986), 961-982.

[23] - Random Walks on Infinite Graphs and Groups (Cambridge University Press, Cambridge, 2000).

University of Technology Graz

Institut für Mathematische Strukturtheorie (Math. C)

Steyrergasse 30

A-8010 Graz

Austria

e-mail: gilch@TUGraz.at 